\title{
Solution of the convex programming problem via second order differential equation system
}

\author{
T. Hajba and M. Kovács
}




\title{
SOLUTION OF THE CONVEX PROGRAMMING PROBLEM VIA SECOND ORDER DIFFERENTIAL EQUATION SYSTEM
}

\author{
T. HAJBA AND M. KOVÁCS \\ Received 16 February, 2011
}

\begin{abstract}
In this paper we investigate a family of second order differential equations which can be considered as the continuous versions of the Fletcher-Reeves iteration for minimization applied to a regularized and penalized convex programming problem. We show that synchronizing the parameter functions of the differential equation the stationary limit point of the trajectories is the minimal norm solution of the given convex programming problem.
\end{abstract}

2000 Mathematics Subject Classification: Primary 34D05; Secondary 90C25; 65K05

Keywords: second order differential equation, minimizer trajectory, stationary point in limit, Lyapunov-type methods, convex programming, Fletcher-Reeves iteration

\section{INTRODUCTION}

Several types of ordinary differential equation (shortly ODE) systems are known whose trajectories minimize a given function. Some of them arise from physics and technology, others are motivated by iterative numerical methods.

The asymptotic behaviour of their trajectories is studied by lot of mathematicians, physicists and engineers. Some of them deal with either the gradient or the Newton method and model the given method by a system of first order differential equations (shortly FO-ODE) system (e.g. Antipin [3], Attouch and Cominetti [4], Botsaris [7], Brown and Bartholomew-Biggs [9], Cominetti et al. [13], Evtushenko and Zhadan [14], Flåm [16], Hauser and Nedić [21], Branin and Hoo [8], Kovács [22,23], (Vasil'ev and Kovács [28]), Venec and Rybashov [31], Zhang et al [32] etc.).

There are some ODE models in connection with the optimization that arise from physical problems such as e.g. the heavy ball with friction, the problem of a nonlinear oscillator with dumping, evolution equations with linear dumping and convex potential. These problems generally lead to a second order differential equations (shortly SO-ODE) system. Results concerning such type of SO-ODE models can be found e.g. in the papers Alvarez [1]; Attouch [2], Attouch et al. [6], Cabot et al. [11,12]), Goudou and Munier [17], Vasil'ev et al. [27], Vasil'ev and Nedich [29]. There are some papers discussing higher order methods (e.g. Vasiljev and Nedić [30], Vasil'ev 
and Nedić [29], Nedić [24]), too. However, the mentioned papers, except Vasil'ev et al. [27], deal with such SO-ODE systems that are linear in $\dot{\mathbf{x}}$.

From the mentioned methods only a few deal with the solution of constrained optimization problem. The authors of the papers Antipin [3], Nedić [24], Vasil'ev and Nedich [29], Vasiljev and Nedić [30] use the projection gradient for handing the constraints, but this technique makes it possible to use only relatively simple constraints. In Kovács [22] the penalty function technique is used and in Vasiljev and Nedić [30], Vasil'ev and Nedich [29] and Vasil'ev et al. [27] the two techniques are used together.

In the cases when the minimum point is not unique, a suitable regularization appears in the ODE systems to control the trajectories to the direction of a well-defined solution (see e.g. in Attouch and Czarnecki [5], Cabot [10], Cominetti et al. [13], Kovács [22], Vasil'ev et al [27] etc.)

It can be seen, that the higher the order of the optimizer ODE system is and more optimization and control parameters are built into the ODE system, the more complicated the synchronization of the conditions between the parameter functions.

In this paper we deal with a SO-ODE system of four parameter functions, two of them define the structure of the SODE system, one is the penalty parameter and one is the control parameter of the regularization.

\section{MATHEMATICAL PRELIMINARIES}

In this section we formulate our convex programming problem (shortly $\mathrm{CP}$ problem) for that we try to associate SO-ODE systems whose trajectories' limit points give the solution of the $\mathrm{CP}$ problem. We describe those known techniques, methods and lemmas that will be used in our investigation.

\subsection{CP problem}

Let $f: \mathbb{R}^{n} \rightarrow \mathbb{R}$ and $g_{i}: \mathbb{R}^{n} \rightarrow \mathbb{R}, i=1, \ldots, m$ be convex continuously differentiable functions and let $g_{i}: \mathbb{R}^{n} \rightarrow \mathbb{R}, i=m+1, \ldots, s$ be linear ones, and let us consider the following $\mathrm{CP}$ problem:

$$
f(\mathbf{x}) \rightarrow \min _{\mathbf{x} \in X}
$$

where

$$
X=\left\{\mathbf{x} \in \mathbb{R}^{n}: g_{i}(\mathbf{x}) \leq 0, i=1, \ldots, m, g_{i}(\mathbf{x})=0, i=m+1, \ldots, s .\right\}
$$

We assume that the problem (2.1)-(2.2) satisfies the following conditions:

A1): it is Slater-regular, i.e. there exists a Slater-point $\overline{\mathbf{x}}$ such that $g_{i}(\overline{\mathbf{x}})<0$, $i=1, \ldots, m$,

A2): the minimum is finite, i.e. $f_{*}=\inf f(\mathbf{x})>-\infty$, and the given CP problem has at least one solution, i.e. $X_{*}=\left\{\mathbf{x} \in X: f(\mathbf{x})=f_{*}\right\} \neq \varnothing$. 
The constrained optimization problem may be solved by solving a sequence of unconstrained minimization problems

$$
\Phi_{k}(\mathbf{x})=f(\mathbf{x})+A_{k} P(\mathbf{x}) \rightarrow \min _{\mathbf{x} \in \mathbb{R}^{n}}, \quad k=1,2, \ldots,
$$

where the penalty function $P(\mathbf{x})$ is defined by

$$
P(\mathbf{x})=\frac{1}{p} \sum_{i=1}^{s}\left|g_{i}^{+}(\mathbf{x})\right|^{p}, \quad\left|g_{i}^{+}(\mathbf{x})\right|= \begin{cases}\max \left(0, g_{i}(\mathbf{x})\right), & i=1, \ldots, m \\ \left|g_{i}(\mathbf{x})\right|, & i=m+1, \ldots, s\end{cases}
$$

and $p>1$, moreover $A_{k} \rightarrow \infty$ if $k \rightarrow \infty$. If $\mathbf{x}_{k}^{*}$ is a minimum point of the $k$-th problem $\Phi_{k}(\mathbf{x}) \rightarrow \min _{\mathbf{x} \in \mathbb{R}^{n}}$ then there is a subsequence $\left\{\mathbf{x}_{k_{j}}\right\}$ of $\left\{\mathbf{x}_{k}\right\}$ which converges to one of the minimum point of the original problem (2.1)-(2.2) (see Fiacco and McCormick [15]).

However from the convexity of the objective and constraints functions it does not follow that the minimum point of the CP problem is unique, and hence it can not be expected that any subsequence tends to the same minimum point. Our aim is to find the so called minimal norm solution, i.e. we seek the point $\mathbf{x} \in X_{*}$ such that

$$
\left\|\mathbf{x}_{*}\right\|=\inf _{x \in X_{*}}\|\mathbf{x}\| .
$$

Therefore we use the regularization technique of A. N. Tichonov (see Tichonov [26], Tikhonov and Vasilev [25]).

The regularization of the convex programming means that the penalized minimizing function will be approximated with a bundle of strong convex functions depending on a parameter of the regularization. By harmonizing the chosen regularization parameter with the coefficient of the penalty function, one can expect that the sequence of the unique minimum points of the auxiliary functions tends to one of the well defined minimum point of the original minimizing function independently of the starting point. The possibility of this type of regularization is based on Lemma 1. It simplifies the Theorem of (Vasil'ev and Kov ács [28]) in the following sense: the used topological space is $\mathbb{R}^{n}$ with the norm-topology, all constraints are handled by penalty function of type (2.4), $\Omega(\mathbf{x})=\|\mathbf{x}\|^{2}$. Moreover, it is also assumed that the objective and constraints functions values as well as the unconstrained minimum can be computed exactly.

Lemma 1. Let the CP-problem (2.1)-(2.2) satisfy the conditions A1-A2 and let $\mathbf{x}_{*}$ denote the minimal norm solution of this problem. Let us introduce the bundle of functions

$$
T_{k}(\mathbf{x})=f(\mathbf{x})+A_{k} P(\mathbf{x})+\frac{1}{2} \lambda_{k}\|\mathbf{x}\|^{2}, k=1,2, \ldots,
$$

where $P(\mathbf{x})$ is defined by (2.4), $\left\{A_{k}: k=0,1,2, \ldots\right\}$ is an infinite monotone increasing and $\left\{\lambda_{k}: k=0,1,2, \ldots\right\}$ is an infinite monotone decreasing sequence satisfying the 
conditions

$$
\lim _{k \rightarrow \infty} A_{k}=\infty, \quad \lim _{k \rightarrow \infty} \lambda_{k}=0, \quad \lim _{k \rightarrow \infty} A_{k}^{1 /(p-1)} \lambda_{k}=\infty .
$$

If $\mathbf{x}_{k}^{*}$ denotes the unique solution of the unconstrained minimization problem $T_{k}(\mathbf{x}) \rightarrow$ $\min _{\mathbf{x} \in \mathbb{R}^{n}}$ with fixed $k$, then the sequence $\left\{\left\|x_{k}^{*}\right\|, k=0,1, \ldots\right\}$ is bounded and

$$
\lim _{k \rightarrow \infty} f\left(\mathbf{x}_{k}^{*}\right)=f_{*} ; \quad \lim _{k \rightarrow \infty} A_{k} P\left(\mathbf{x}_{k}^{*}\right)=0 ; \quad \lim _{k \rightarrow \infty}\left\|\mathbf{x}_{k}^{*}-\mathbf{x}_{*}\right\|=0 .
$$

This lemma remains valid if the parameter sequences are changed for continuous functions of time $t$ and everywhere in the lemma the convergence is considered as $t \rightarrow \infty$.

In our investigation the continuous version of this lemma will be used.

\subsection{ODE systems associated to $C P$ problems}

The most frequently used methods of unconstrained optimization are iterative methods, e.g. the gradient and Newton methods, the Fletcher-Reeves iteration etc. Any iterative method can be considered as Euler-discretization of an ODE system with stepsize 1. This ODE system is called the continuous version of the given method. So, with different numerical methods one can associate several types of differential equations with the same $\mathrm{CP}$ problem and one can define those conditions, under which the trajectories of these ODE systems converge to a minimum point. The associated ODE system may be first or higher order.

To speak about the limit point of the trajectories if $t \rightarrow \infty$ it is necessary that the right maximal interval will be the whole $\left[t_{0}, \infty\right)$. To guarantee this property we will use the idea of local Lipschitz continuity.

We will say that the vector function $\Phi: W \rightarrow \mathbb{R}^{k}$ defined on the open subset $W \subseteq \mathbb{R}^{n}$ is local Lipschitz continuous if it is Lipschitz continuous on every compact subset of $W$. If $I_{0} \subset \mathbb{R}$ is open, then the function $\Phi(\mathbf{x}, t): W \times I_{0} \rightarrow \mathbb{R}^{k}$ is local Lipschitz continuous if it is Lipschitz continuous in $\mathbf{x}$ on any compact subset $V \subset W$, and continuous in $t$ on any closed interval $I \subset I_{0}$.

If an ODE system associated with an iterative method of minimization has a unique solution on $\left[t_{0}, \infty\right)$ then we say that

- it is a minimizing model if along its trajectories $\lim _{t \rightarrow \infty} f(\mathbf{x}(t))=f_{*}$;

- it is convergent if any trajectory of it converges in norm to some $\mathbf{x}_{*} \in X_{*}$, i.e. $\left\|\mathbf{x}(t)-\mathbf{x}_{*}\right\| \rightarrow 0$.

The trajectories of a convergent minimizing model are called minimizing trajectories.

The ODE systems associated with an unconstrained optimization problem $f(\mathbf{x}) \rightarrow$ $\min _{\mathbf{x} \in \mathbb{R}^{n}} f(\mathbf{x})$ by gradient and Newton methods can be written in the forms

$$
\begin{array}{ll}
\dot{\mathbf{x}}=-\nabla f(\mathbf{x}), & \dot{\mathbf{x}}=-\mathbf{H}(\mathbf{x})^{-1} \nabla f(\mathbf{x}) \\
\mathbf{x}\left(t_{0}\right)=\mathbf{x}_{0} & \mathbf{x}\left(t_{0}\right)=\mathbf{x}_{0},
\end{array}
$$


respectively, where $\mathbf{H}(\mathbf{x})$ is the Hessian of $f(\mathbf{x})$. In these cases if $\mathbf{x}_{*}$ denotes a minimum point of the function $f(\mathbf{x})$ then $\mathbf{x}_{*}$ is a stationary point of both FO-ODE system.

In general, $\mathbf{x}_{*}$ is a stationary point for a FO-ODE system if its right hand side turns into a null-vector in this point for all $t \geq t_{0}$. If the minimum point is a stationary point of the associated ODE system then the convergence of the trajectories to $\mathbf{x}_{*}$ is equivalent with the asymptotic stability of $\mathbf{x}_{*}$, therefore the well known Lyapunov function methods are useful to prove the convergence with an appropriately chosen Lyapunov function (see e.g. Evtushenko and Zhadan [14], Flåm [16], Venec and Rybashov [31]).

In contrary, in a FO-ODE system associated with a regularized unconstrained or a regularized convex programming problem given by (2.1)-(2.2) where the constraints are handled by penalty function of type (2.4) the minimum point $\mathbf{x}_{*}$ is not necessary stationary point (e.g. in the case of regularized continuous gradient method).

As it was pointed out in Kovács [23], the minimization models modelled by FOODE systems can be divided into two classes. Those models described by a FO-ODE system for which the point $\mathbf{x}_{*}$ is a stationary point of the system belong to the first class. To the second class of the models belong those continuous first order models, for which the minimum point is not stationary, but the trajectories exist on the whole $\left[t_{0}, \infty\right)$ and along the trajectories the right hand side vector of the differential equation system tends to the null-vector if $t \rightarrow \infty$. Following Kovács [23] we say in this case, that $\mathbf{x}_{*}$ is stationary in limit.

The definition of stationary and stationary in limit points can be extended for higher order ODE systems, too. We will say, that a point is stationary point or stationary in limit point of a second or higher order ODE system if it is stationary or stationary in limit point respectively for the equivalent FO-ODE system.

As it was shown in Kovács [22,23] the Lyapunov-type methods are also applicable to prove the convergence of the trajectories to a point stationary in limit.

The following lemma will be useful in proving the existence of the trajectories of the examined SO-SODE system on $\left[t_{0}, \infty\right)$.

Lemma 2. Suppose that there exist $0 \leq T_{0} \leq T_{\max }<\infty$ such that

(1) the parametric scalar functions $g(t, \tau), \tau \in\left(T_{0}, T_{\max }\right]$ are defined and nonnegative for all $t \in\left[T_{0}, \tau\right)$, they are continuously differentiable with respect to $t$;

(2) $g\left(T_{0}, \tau\right) \leq K$ uniformly in $\tau$;

(3) for every fixed $\tau \in\left(T_{0}, T_{\max }\right]$ the function $g(t, \tau)$ satisfies the differential inequality

$$
\frac{\mathrm{d}}{\mathrm{d} t} g(t, \tau) \leq-\varphi(t) g(t, \tau)+\psi(t)(\tau-t)^{k}
$$

on the interval $\left[T_{0}, \tau\right)$, where $k$ is a nonnegative integer, the functions $\varphi(t)>$ 0 and $\psi(t)$ are defined and continuous on $\left[T_{0}, \infty\right)$ and integrable on any finite interval of $\left[T_{0}, \infty\right)$. 
Then for every fixed $\tau \in\left(T_{0}, T_{\max }\right]$ the function $g(t, \tau)$ is bounded on $\left[T_{0}, \tau\right)$ and $g(\tau, \tau)=\lim _{t \rightarrow \tau} g(t, \tau)<\infty$.

Proof. Let

$$
u(t, \tau)=g\left(T_{0}, \tau\right) \mathrm{e}^{-\int_{T_{0}}^{t} \varphi(v) \mathrm{d} v}+\int_{T_{0}}^{t} \psi(\theta)(\tau-\theta)^{k} \mathrm{e}^{\int_{T_{0}}^{\theta} \varphi(v) \mathrm{d} v} \mathrm{~d} \theta
$$

be the solution of the linear ODE

$$
\dot{u}=-\varphi(t) u+\psi(t)(\tau-t)^{k}, \quad u\left(T_{0}, \tau\right)=g\left(T_{0}, \tau\right) .
$$

Since $\varphi(t)$ and $\psi(t)$ are continuous on $\left[T_{0}, \infty\right), u(t, \tau)$ is the unique solution on $\left[T_{0}, \infty\right)$, consequently $u(t, \tau)$ is bounded on every closed finite interval of $\left[T_{0}, \infty\right)$.

Using the Gronwall lemma we have that for every $\tau \in\left(T_{0}, T_{\max }\right]$

$$
g(t, \tau) \leq u(t, \tau) \text { for all } T_{0} \leq t<\tau,
$$

from this fact and from the continuity of $g(t, \tau)$ the boundedness of $g(t, \tau)$ on $\left[T_{0}, \tau\right]$ immediately follows.

To examine the limit points of the trajectories when the maximal right hand interval of existence is $\left[t_{0}, \infty\right)$, the following lemma taken from Kovács [23] will be useful. However, we have to remark that detailed proof of this statement can only be found in the proof of the Theorem of Kovács [22], and only for the special case $k=1$. Therefore, here we sketch its proof, too.

Lemma 3. Let the conditions 1-3 of the Lemma 2 be satisfied when $T_{\max }=\infty$. Moreover assume that the functions $\varphi(t)$ and $\psi(t)$ are endowed with the following properties:

(4) $\int_{T_{0}}^{\infty} \varphi(t) d t=\infty, \quad \lim _{t \rightarrow \infty} \frac{\psi(t)}{\varphi^{k+1}(t)}=0$;

(5) in the case $k \geq 1$ the function $\varphi(t)$ is differentiable and $\lim _{t \rightarrow \infty} \frac{\dot{\varphi}(t)}{\varphi^{2}(t)}=0$, then $\lim _{\tau \rightarrow \infty} g(\tau, \tau)=0$.

Proof. It is enough to prove that $u(\tau, \tau) \rightarrow \infty$ if $t \rightarrow \infty$ where $u(t, \tau)$ is defined by (2.8). Under the condition 3 . the convergence of the first term of (2.8) to zero is trivial. By induction on $k$ it can be proved that for all nonnegative integer $k$ the limit $\lim _{\tau \rightarrow \infty} \mathrm{e}^{\int_{T_{0}}^{\tau} \varphi(v) \mathrm{d} v} \varphi^{k}(\tau)=\infty$ holds true and hence we can estimate the second term by applying $(k+1)$ times the L'Hospital rule and finally by the additional conditions 4 and 5 of this lemma.

The function $g(t, \tau)$ plays the role of a Lyapunov-like function in stability theory or the energy function in the theory of dissipative systems. 


\section{MINIMIZING PROPERTY OF THE REGULARIZED FR-TYPE SO-ODE}

(R-FR-TYPE SO-ODE) SYSTEM ASSOCIATED WITH THE CP PROBLEM

3.1. Construction of the R-FR-type SO-ODE system associated with the CP problem

Let us now examine an ODE system associated with the CP problem (2.1)-(2.2) by the Fletcher-Reeves iteration applied to the minimization of the penalized and regularized unconstrained function (2.6). This iteration can be formulated as follows: Let be given two vectors, namely $\mathbf{x}_{k} \in \mathbb{R}^{n}$ and $\mathbf{d}_{k} \in \mathbb{R}^{n}$ which are called the $k$-th state vector and $k$-th searching direction, respectively. Let the sequence $\left(\mathbf{x}_{k}, \mathbf{d}_{k}\right) \in \mathbb{R}^{n} \times \mathbb{R}^{n}$ be defined by

$$
\begin{aligned}
& \mathbf{x}_{k+1}=\mathbf{x}_{k}+\alpha_{k} \mathbf{d}_{k}, \\
& \mathbf{d}_{k+1}=-\nabla_{\mathbf{x}} F\left(x_{k+1}\right)+\beta_{k} \mathbf{d}_{k}, \quad k=0,1,2, \ldots,
\end{aligned}
$$

starting with $\mathbf{x}_{0} \in \mathbb{R}^{n}, \mathbf{d}_{0}=-\nabla_{\mathbf{x}} F_{k}\left(\mathbf{x}_{0}\right)$. To obtain a convergent process we have to use well defined (we do not present here the details) changing rules for the sequences $\alpha_{k}, \beta_{k}, A_{k}$ and $\lambda_{k}$.

It is easy to see that this iteration can be considered as the Euler discretization of the non-autonomous FO-ODE system of $2 n$ variables

$$
\begin{aligned}
& \dot{\mathbf{x}}=\alpha(t) \mathbf{d} \\
& \dot{\mathbf{d}}=-\nabla_{\mathbf{x}} F(\mathbf{x}+\alpha(t) \mathbf{d}, t)+(\beta(t)-1) \mathbf{d}
\end{aligned}
$$

were

$$
F(\mathbf{x}, t)=f(\mathbf{x})+A(t) P(\mathbf{x})+\frac{1}{2} \lambda(t)\|\mathbf{x}\|^{2},
$$

corresponds to (2.6) and the rules of change of all parameters are described by continuous functions.

(3.3)-(3.4) can be transformed by the substitution $\mathbf{p}(t)=\alpha(t) \mathbf{d}(t)$ into the following ones:

$$
\begin{aligned}
\dot{\mathbf{x}} & =\mathbf{p} \\
\dot{\mathbf{p}} & =-\alpha(t) \nabla_{\mathbf{x}} F(\mathbf{x}+\mathbf{p}, t)-\gamma(t) \mathbf{p}
\end{aligned}
$$

with the initial values

$$
\mathbf{x}\left(t_{0}\right)=\mathbf{x}_{0}, \mathbf{p}\left(t_{0}\right)=\mathbf{p}_{0},
$$

where $\gamma(t)=-\frac{\dot{\alpha}(t)}{\alpha(t)}-\beta(t)+1$.

It is clear, that the FO-ODE system (3.6)-(3.7) with the initial values (3.8) is equivalent to the SO-ODE system

$$
\ddot{\mathbf{x}}+\gamma(t) \dot{\mathbf{x}}+\alpha(t) \nabla_{\mathbf{x}} F(\mathbf{x}+\dot{\mathbf{x}}, t)=0
$$

with initial values

$$
\mathbf{x}\left(t_{0}\right)=\mathbf{x}_{0}, \dot{\mathbf{x}}\left(t_{0}\right)=\mathbf{p}_{0} .
$$


The ODE systems given by (3.6),(3.7) and (3.8) or by (3.9) and (3.10) will be called regularized FR-type SO-ODE (shortly R-FR type SO-ODE) system associated with the CP problem.

Since the Fletcher-Reeves iteration uses the new state point in the construction of the new direction, our SO-ODE system usually will not be linear in the first derivative vector $\dot{\mathbf{x}}$ in contrary to the examined SO-ODE systems mentioned in the Introduction. In connection with optimization, such type of SO-ODE systems has been investigated in Hajba [18] for the unconstrained minimization of a strong convex function. The asymptotic behaviour of the regularized FR-type SO-ODE system associated with the unconstrained minimization of convex but not necessary strong convex function has been analyzed in Hajba [20]. A similar problem for constrained optimization has been examined in Vasil'ev et al. [27], but their result is obtained under other (and more complicated) assumptions than the ones used here.

It is worth looking at the special case of the CP problem when the objective function is quadratic and the constraints are given with a linear equality system, i.e. a hyperspace. Namely, let $\mathbf{Q}$ be a symmetric positive semidefinite $n \times n$ matrix and $\mathbf{c} \in \mathbb{R}^{n}$. Moreover, let be given an $m \times n$ matrix $\mathbf{B}$, and a vector $\mathbf{b}_{0} \in \mathbb{R}^{m}$. Consider the $\mathrm{CP}$ problem

$$
\frac{1}{2}\langle\mathbf{x}, \mathbf{Q x}\rangle+\langle\mathbf{c}, \mathbf{x}\rangle \quad \text { subject to } \quad \mathbf{B} \mathbf{x}=\mathbf{b}_{0} .
$$

To define the function $F(\mathbf{x}, t)$ we use the penalty function

$$
P(\mathbf{x})=\frac{1}{2}\left\|\mathbf{B} \mathbf{x}-\mathbf{b}_{0}\right\|^{2} .
$$

Then the SO-ODE system (3.9) takes the following shape

$$
\ddot{\mathbf{x}}+(\gamma(t) \mathbf{E}+\mathbf{H}(t)) \dot{\mathbf{x}}+\mathbf{H}(t) \mathbf{x}+\mathbf{h}(t)=\mathbf{0},
$$

with matrix functions

$$
\mathbf{H}(t)=\alpha(t)\left(\mathbf{Q}+A(t) \mathbf{B}^{T} \mathbf{B}+\lambda(t) \mathbf{E}\right), \quad \mathbf{h}(t)=\alpha(t)\left(\mathbf{c}+A(t) \mathbf{B}^{T} \mathbf{b}_{0}\right),
$$

where $\mathbf{E}$ denotes the unit matrix and the upper index $T$ denotes transposition.

Although in this special case the SO-ODE system is linear in $\dot{\mathbf{x}}$, too, but its coefficient is a time dependent matrix function. So, our SO-ODE system for this relatively simple problem also essentially differs from the SO-ODE systems analized in papers mentioned in the Introduction, and it has not been examined yet.

\subsection{Asymptotic behaviour of the trajectories of the R-FR-type SO-ODE systems}

The following proposition characterizes the R-FR-type SO-ODE systems associated with the CP problem given by (2.1)-(2.2). 
Proposition 1. Let us assume that

(1) the CP-problem defined by (2.1)-(2.2) satisfies the conditions $\mathbf{A 1}$ and $\mathbf{A 2}$ and the functions $f(\mathbf{x})$ and $g_{i}(\mathbf{x}), i=1, \ldots, m$ are continuously differentiable convex functions on $\mathbb{R}^{n}$ and the gradients $\nabla f(\mathbf{x})$ and $\nabla g_{i}(\mathbf{x}), i=1, \ldots, m$ are local Lipschitz continuous;

(2) the penalty function $P(\mathbf{x})$ in the construction of $F(\mathbf{x}, t)$ is defined by (2.4);

(3) the parameter functions $\alpha(t)$ and $\gamma(t)$ of the systems (3.6)-(3.7) and (3.9) fulfill the following conditions:

a) $\alpha(t)$ is a positive, monotone decreasing and continuously differentiable function on $\left[t_{0}, \infty\right)$;

b) $\gamma(t)$ is a monotone nonincreasing, continuously differentiable function on $\left[t_{0}, \infty\right)$ and $\gamma(t) \geq \gamma_{\infty}>1$ for all $t \geq t_{0}$;

(4) the coefficient $A(t)$ of the penalty function in the construction of $F(\mathbf{x}, t)$ is a positive continuously differentiable concave function and satisfies the conditions $\lim _{t \rightarrow \infty} A(t)=\infty$ and $\alpha(t) A(t) \leq A_{0}<\infty ;$

(5) for the regularization parameter $\lambda(t)$ the following assumptions hold:

a) $\lambda(t)$ is a positive continuously differentiable monotone decreasing convex function on $\left[t_{0}, \infty\right)$ for all $t \geq t_{0}$ and $\lim _{t \rightarrow \infty} \lambda(t)=\lim _{t \rightarrow \infty} \dot{\lambda}(t)=0$;

b) $\alpha\left(t_{0}\right) \lambda\left(t_{0}\right)=1$, while $\int_{t_{0}}^{\infty} \alpha(t) \lambda(t)=\infty$;

c) $\lim _{t \rightarrow \infty} \frac{\dot{\alpha}(t)}{\alpha^{2}(t) \lambda(t)}=\lim _{t \rightarrow \infty} \frac{\dot{\lambda}(t)}{\alpha(t) \lambda^{2}(t)}=\lim _{t \rightarrow \infty} \frac{\dot{A}(t)}{\alpha(t) \lambda^{2}(t)}=0$

d) $\lim _{t \rightarrow \infty} A(t)^{1 /(p-1)} \lambda(t)=\infty$.

Then

(1) the trajectories of (3.6)-(3.7), respectively of (3.9) exist and unique on the whole half-line $\left[t_{0}, \infty\right)$ with any initial point (3.8) resp. (3.10);

(2) the trajectories converge to the minimal norm solution, i.e. if $\mathbf{x}_{*}$ satisfies the condition $\inf _{\mathbf{x} \in X_{*}}\|\mathbf{x}\|=\left\|\mathbf{x}_{*}\right\|$, then $\lim _{t \rightarrow \infty}\left\|\mathbf{x}(t)-\mathbf{x}_{*}\right\|=0$;

(3) $\lim _{t \rightarrow \infty} f(\mathbf{x}(t))=f\left(\mathbf{x}_{*}\right)=\inf _{\mathbf{x} \in X} f(\mathbf{x})$, i.e. the SO-ODE system given by (3.6)(3.7) or (3.9) is a minimizing model and its trajectories are minimizing trajectories;

(4) $\lim _{t \rightarrow \infty}\|\mathbf{p}(t)\|=\lim _{t \rightarrow \infty}\|\dot{\mathbf{x}}(t)\|=0$;

(5) $\lim _{t \rightarrow \infty}\|\ddot{\mathbf{x}}(t)\|=0$, i.e. the minimal norm solution $\mathbf{x}_{*}$ is a stationary in limit minimum point.

Proof. The continuity in $t$ of the right hand side of (3.6)-(3.7), resp. (3.9) immediately follows from the continuity conditions given for the parameter functions. From the convexity and continuity of $g_{i}(\mathbf{x}), i=1, \ldots, m$ follows the local Lipschitz 
continuity of these functions which together with the assumed local Lipschitz continuity of $\nabla g_{i}(\mathbf{x}), i=1, \ldots, m$ calls forth the local Lipschitz continuity of $\nabla P(\mathbf{x})$. Consequently the right hand side of (3.6)-(3.7) is also local Lipschitz continuous in $\mathbf{x}$. This property guaranties the existence and uniqueness of the trajectories on a right maximal interval $\left[t_{0}, t_{\max }\right)$ with $t_{0}<t_{\max } \leq \infty$. In order to prove that $t_{\max }=\infty$, let us show that $|\dot{\mathbf{x}}(t)|$ and $|\ddot{\mathbf{x}}(t)|=|\dot{\mathbf{p}}(t)|$ are bounded.

For every fixed $t_{0}<\tau<\infty$ the function $F(\mathbf{x}, \tau)$ defined by (3.5) is a strong convex function, therefore it has a unique minimum point $\mathbf{x}_{\tau}^{*}$. Let $\mathbf{x}_{*}$ be the minimal norm solution of the CP-problem. Under the assumption 4, 5.a) and 5.d) of the proposition the conditions of the Lemma 1 are satisfied, so there exists a constant $K$ such that $\left\|x_{\tau}^{*}\right\| \leq K$ for all $t_{0} \leq \tau$.

Let us introduce the parametric function

$$
\begin{aligned}
g(t, \tau)= & \frac{1}{2}\left\|\mathbf{x}(t)-\mathbf{x}_{\tau}^{*}+\mathbf{p}(t)\right\|^{2}+ \\
& +\frac{1}{4} \alpha(t) \lambda(t)\left\|\mathbf{x}(t)-\mathbf{x}_{\tau}^{*}\right\|^{2}+\frac{1}{2}(\gamma(t)-1)\left\|\mathbf{x}(t)-\mathbf{x}_{\tau}^{*}\right\|^{2}
\end{aligned}
$$

for fixed $t_{\max } \geq \tau \geq t_{0}$.

It follows from the conditions 3.a), 3.b) and 5.a) that $g(t, \tau) \geq 0$ for all $t_{0} \leq t<\tau$, and Lemma 1 guaranties the uniformly boundedness of $g\left(t_{0}, \tau\right)$ in $\tau$.

For the derivative of $g(t, \tau)$ we have

$$
\begin{aligned}
\frac{\mathrm{d}}{\mathrm{d} t} g(t, \tau)= & -\alpha(t)\left\langle\nabla_{\mathbf{x}} F(\mathbf{x}(t)+\mathbf{p}(t), t), \mathbf{x}(t)-\mathbf{x}_{\tau}^{*}+\mathbf{p}(t)\right\rangle+ \\
& +\frac{1}{4} \frac{\mathrm{d}}{\mathrm{d} t}(\alpha(t) \lambda(t))\left\|\mathbf{x}(t)-\mathbf{x}_{\tau}^{*}\right\|^{2}+\frac{1}{2} \alpha(t) \lambda(t)\left\langle\mathbf{x}(t)-\mathbf{x}_{\tau}^{*}, \mathbf{p}(t)\right\rangle+ \\
& +(1-\gamma(t))\|\mathbf{p}(t)\|^{2}+\frac{1}{2} \dot{\gamma}(t)\left\|\mathbf{x}(t)-\mathbf{x}_{\tau}^{*}\right\|^{2}
\end{aligned}
$$

for all $t_{0} \leq t<\tau$. (Here and in the following $\langle\cdot, \cdot\rangle$ denotes the scalar product.)

Omitting the non-positive terms we have that

$$
\begin{aligned}
\frac{\mathrm{d}}{\mathrm{d} t} g(t, \tau) \leq & -\alpha(t)\left\langle\nabla_{\mathbf{x}} F(\mathbf{x}(t)+\mathbf{p}(t), t), \mathbf{x}(t)-\mathbf{x}_{\tau}^{*}+\mathbf{p}(t)\right\rangle+ \\
& +\frac{1}{2} \alpha(t) \lambda(t)\left\langle\mathbf{x}(t)-\mathbf{x}_{\tau}^{*}, \mathbf{p}(t)\right\rangle= \\
= & -\alpha(t)\left\langle\nabla_{\mathbf{x}} F(\mathbf{x}(t)+\mathbf{p}(t), t)-\nabla_{\mathbf{x}} F\left(\mathbf{x}_{\tau}^{*}, t\right), \mathbf{x}(t)-\mathbf{x}_{\tau}^{*}+\mathbf{p}(t)\right\rangle- \\
& -\alpha(t)\left\langle\nabla_{\mathbf{x}} F\left(\mathbf{x}_{\tau}^{*}, t\right)-\nabla_{\mathbf{x}} F\left(\mathbf{x}_{\tau}^{*}, \tau\right), \mathbf{x}(t)-\mathbf{x}_{\tau}^{*}+\mathbf{p}(t)\right\rangle+ \\
& +\frac{1}{2} \alpha(t) \lambda(t)\left\langle\mathbf{x}(t)-\mathbf{x}_{\tau}^{*}, \mathbf{p}(t)\right\rangle .
\end{aligned}
$$


For every fixed $t$ the convexity modulus of the strong convex function $F(\mathbf{x}, t)$ is $\frac{1}{2} \lambda(t)$, therefore

$$
\begin{aligned}
-\alpha(t)\left\langle\nabla_{\mathbf{x}} F(\mathbf{x}(t)+\mathbf{p}(t), t)\right. & \left.-\nabla_{\mathbf{x}} F\left(\mathbf{x}_{\tau}^{*}, t\right), \mathbf{x}(t)-\mathbf{x}_{\tau}^{*}+\mathbf{p}(t)\right\rangle \leq \\
& \leq-\alpha(t) \lambda(t)\left\|\mathbf{x}(t)-\mathbf{x}_{\tau}^{*}+\mathbf{p}(t)\right\|^{2} .
\end{aligned}
$$

To estimate the term $-\alpha(t)\left\langle\nabla_{\mathbf{x}} F\left(\mathbf{x}_{\tau}^{*}, t\right)-\nabla_{\mathbf{x}} F\left(\mathbf{x}_{\tau}^{*}, \tau\right), \mathbf{x}(t)-\mathbf{x}_{\tau}^{*}+\mathbf{p}(t)\right\rangle$ we apply the inequality

$$
-\langle\mathbf{a}, \mathbf{b}\rangle \leq \frac{1}{\lambda(t)}\|\mathbf{a}\|^{2}+\frac{\lambda(t)}{4}\|\mathbf{b}\|^{2} .
$$

Namely,

$$
\begin{gathered}
-\alpha(t)\left\langle\nabla_{\mathbf{x}} F\left(x_{\tau}^{*}, t\right)-\nabla_{\mathbf{x}} F\left(x_{\tau}^{*}, \tau\right), \mathbf{x}(t)-\mathbf{x}_{\tau}^{*}+\mathbf{p}(t)\right\rangle= \\
=-\alpha(t)\left\langle(A(t)-A(\tau)) \nabla P\left(\mathbf{x}_{\tau}^{*}\right), \mathbf{x}(t)-\mathbf{x}_{\tau}^{*}+\mathbf{p}(t)\right\rangle- \\
-\alpha(t)\left\langle(\lambda(t)-\lambda(\tau)) \mathbf{x}_{\tau}^{*}, \mathbf{x}(t)-\mathbf{x}_{\tau}^{*}+\mathbf{p}(t)\right\rangle \leq \\
\leq \frac{\alpha(t)}{\lambda(t)}\left((A(t)-A(\tau))^{2}\left\|\nabla P\left(\mathbf{x}_{\tau}^{*}\right)\right\|^{2}+(\lambda(t)-\lambda(\tau))^{2}\left\|\mathbf{x}_{\tau}^{*}\right\|^{2}\right)+ \\
+\frac{\alpha(t) \lambda(t)}{2}\left\|\mathbf{x}(t)-\mathbf{x}_{\tau}^{*}+\mathbf{p}(t)\right\|^{2}
\end{gathered}
$$

Since the sequence $\left\|\mathbf{x}_{\tau}^{*}\right\|$ is bounded due to the Lemma 1 and $\|\nabla P(\mathbf{x})\|$ is continuous, therefore there exists a constant $K \geq 0$ do not depending on the parameter $\tau$ such that $\max \left\{\left\|\nabla P\left(\mathbf{x}_{\tau}^{*}\right),\right\| \mathbf{x}_{\tau}^{*} \|\right\} \leq K^{\frac{1}{2}}$. Using this fact and Conditions 4. and 5.a we have that

$$
\begin{aligned}
-\alpha(t) & \left\langle\nabla_{\mathbf{x}} F\left(\mathbf{x}_{\tau}^{*}, t\right)-\nabla_{\mathbf{x}} F\left(\mathbf{x}_{\tau}^{*}, \tau\right), \mathbf{x}(t)-\mathbf{x}_{\tau}^{*}+\mathbf{p}(t)\right\rangle \leq \\
\leq & K \frac{\alpha(t)}{\lambda(t)}\left(\dot{A}^{2}(t)+\dot{\lambda}^{2}(t)\right)(\tau-t)^{2}+\frac{\alpha(t) \lambda(t)}{2}\left\|\mathbf{x}(t)-\mathbf{x}_{\tau}^{*}+\mathbf{p}(t)\right\|^{2}= \\
& =\frac{\alpha(t) \lambda(t)}{2}\left\|\mathbf{x}(t)-\mathbf{x}_{\tau}^{*}+\mathbf{p}(t)\right\|^{2}+\psi(t)(\tau-t)^{2},
\end{aligned}
$$

where $\psi(t)=K \frac{\alpha(t)}{\lambda(t)}\left(\dot{A}^{2}(t)+\dot{\lambda}^{2}(t)\right)$.

Substituting the inequalities (3.12) and (3.13) into (3.11) and taking into consideration that $\gamma(t)$ is non-increasing we have that

$$
\begin{aligned}
\frac{\mathrm{d}}{\mathrm{d} t} g(t, \tau) \leq & -\frac{1}{2} \alpha(t) \lambda(t)\left\|\mathbf{x}(t)-\mathbf{x}_{\tau}^{*}+\mathbf{p}(t)\right\|^{2}+\frac{1}{2} \alpha(t) \lambda(t)\left\langle\mathbf{x}(t)-\mathbf{x}_{\tau}^{*}, \mathbf{p}(t)\right\rangle+ \\
& +\psi(t)(\tau-t)^{2}=
\end{aligned}
$$




$$
\begin{aligned}
= & -\frac{1}{4} \alpha(t) \lambda(t)\left\|\mathbf{x}(t)-\mathbf{x}_{\tau}^{*}+\mathbf{p}(t)\right\|^{2}-\frac{1}{4} \alpha(t) \lambda(t)\left\|\mathbf{x}(t)-\mathbf{x}_{\tau}^{*}+\mathbf{p}(t)\right\|^{2}+ \\
& +\frac{1}{2} \alpha(t) \lambda(t)\left\langle\mathbf{x}(t)-\mathbf{x}_{\tau}^{*}, \mathbf{p}(t)\right\rangle+\psi(t)(\tau-t)^{2} \leq \\
\leq & -\frac{1}{4} \alpha(t) \lambda(t)\left\|\mathbf{x}(t)+\mathbf{p}(t)-\mathbf{x}_{\tau}^{*}\right\|^{2}-\frac{1}{8} \alpha(t) \lambda(t)\left\|\mathbf{x}-\mathbf{x}_{\tau}^{*}\right\|^{2}- \\
& -\frac{1}{8} \alpha(t) \lambda(t) \cdot \frac{\gamma(t)-1}{\gamma\left(t_{0}\right)-1}\left\|\mathbf{x}-\mathbf{x}_{\tau}^{*}\right\|^{2}+\psi(t)(\tau-t)^{2}= \\
= & -a(t) \frac{1}{2}\left\|\mathbf{x}(t)+\mathbf{p}(t)-\mathbf{x}_{\tau}^{*}\right\|^{2}-b(t) \frac{1}{4} \alpha(t) \lambda(t)\left\|\mathbf{x}-\mathbf{x}_{\tau}^{*}\right\|^{2}- \\
& -c(t) \frac{1}{2}(\gamma(t)-1)\left\|\mathbf{x}-\mathbf{x}_{\tau}^{*}\right\|^{2}+\psi(t)(\tau-t)^{2},
\end{aligned}
$$

where $a(t)=\frac{1}{2} \alpha(t) \lambda(t), b(t)=\frac{1}{2}, c(t)=\frac{1}{4\left(\gamma\left(t_{0}\right)-1\right)} \alpha(t) \lambda(t)=C_{1} \alpha(t) \gamma(t)$.

Since $\alpha(t) \lambda(t) \leq 1$, so $a(t) \leq \frac{1}{2}$ for all $t \geq t_{0}$. Otherwise, $c(t)=2 C_{1} a(t)$, consequently, there exists $K_{1}=\min \left\{1,2 C_{1}\right\}>0$, depending only on $\gamma\left(t_{0}\right)$ such that

$$
\frac{\mathrm{d}}{\mathrm{d} t} g(t, \tau) \leq-K_{1} \alpha(t) \lambda(t) g(t, \tau)+\psi(t)(\tau-t)^{2}
$$

for all $t_{0} \leq t<\tau$.

The functions $g(t, \tau), \varphi(t)=K_{1} \alpha(t) \lambda(t)$ and $\psi(t)$ satisfy the conditions 1 and 2 of the Lemma 2, so there exists a constant $K_{g}$ such that $g\left(t, t_{\max }\right) \leq K_{g}$ for all $t_{0} \leq t<t_{\max }$.

Let us assume, that the right maximal interval of existence is finite, i.e. $t_{\max }<\infty$. Then $g\left(t, t_{\max }\right) \leq K_{g}$ for all $t \in\left[t_{0}, t_{\max }\right)$. From the definition of $g(t, \tau)$ immediately follows that

$$
\left\|\mathbf{x}(t)-\mathbf{x}_{t_{\max }}^{*}+\mathbf{p}(t)\right\| \leq S, \quad\left\|\mathbf{x}(t)-\mathbf{x}_{t_{\max }}^{*}\right\| \leq S
$$

with some constant $S$ for all $t \in\left[t_{0}, t_{\max }\right)$. Using the triangle inequality, the monotonity of the parameter functions $\alpha(t)$ and $\lambda(t)$ and the boundedness of $\mathbf{x}_{\tau}^{*}$ obtained from the Lemma 1, one can easily derive constant $R$ such that for

$$
\|\mathbf{x}(t)\| \leq\left\|\mathbf{x}(t)-\mathbf{x}_{t_{\max }}^{*}\right\|+\left\|\mathbf{x}_{t_{\max }}^{*}\right\| \leq R,
$$

and

$$
\|\dot{\mathbf{x}}(t)\|=\|\mathbf{p}(t)\| \leq\left\|\mathbf{x}(t)-\mathbf{x}_{t_{\max }}^{*}+\mathbf{p}(t)\right\|+\left\|\mathbf{x}(t)-\mathbf{x}_{t_{\max }}^{*}\right\| \leq R .
$$

for all $t \in\left[t_{0}, t_{\max }\right)$. Let

$$
B=\left\{(\mathbf{x}, \mathbf{p}, t):\|\mathbf{x}\| \leq R,\|\mathbf{p}\| \leq R,\left|t-t_{0}\right| \leq t_{\max }\right\} .
$$

Using the continuity of the vector-function $\nabla_{\mathbf{x}} F(\mathbf{x}, t)$ and the upper estimate $\sup _{(\mathbf{x}, \mathbf{p}, t) \in B}\left\|\nabla_{\mathbf{x}} F(\mathbf{x}+\mathbf{p}, t)\right\|$, together with the continuity of $\alpha(t), A(t), \lambda(t)$ we have $\|\ddot{\mathbf{x}}(t)\|=\|\dot{\mathbf{p}}\| \leq R$ if $R$ is chosen large enough. 
Since $B$ is a compact set and $\left(\mathbf{x}(t), \mathbf{p}(t), t_{\max }\right) \in B$, applying again the local existence theorem with the initial value $\left(\mathbf{x}\left(t_{\max }\right), \mathbf{p}\left(t_{\max }\right)\right)$, we can extend the maximal solution to a strictly larger interval, which is a clear contradiction.

Consequently, the solution $(\mathbf{x}(t), \mathbf{p}(t))$ of (3.6)-(3.7) exists on the whole halfline $\left[t_{0}, \infty\right)$, so we can speak about the limit points of the trajectories when $t \rightarrow \infty$.

Conditions 5.a), 5.b) and 5.c) ensure that $g(t, \tau)$ satisfies the conditions 4 and 5 of the Lemma 3 and hence $\lim _{\tau \rightarrow \infty} g(\tau, \tau)=0$. From here and from the continuity of the function $f(\mathbf{x})$ and by using the triangle inequality as in the previous part of the proof, we immediately obtain all statements of the proposition.

\subsection{Admissible parameter functions of the minimizing $R$-FR-type SO-ODE system}

Studying the unconstrained minimization problem without regularization we have found several classes of parameter functions (see Hajba [19]) of the SO-ODE system, which satisfy the conditions given in Hajba [18] for the convergence. Since our problem examined in this paper is more complicated, here we give only two parameter classes which satisfy the conditions of the Proposition 1 assuming that $P(\mathbf{x})=\sum_{i=1}^{m}\left|g_{i}^{+}(\mathbf{x})\right|^{2}$.

It can be seen that we have the widest freedom in the choice of the function $\gamma(t)$, since it does not depend on the other parameters, only Condition 3.b) must be satisfied.

Let

$$
\alpha(t)=\alpha_{0}(1+t)^{-\alpha}, \quad \text { and } \quad \lambda(t)=\lambda_{0}(1+t)^{-\lambda} .
$$

Substituting these functions into the limit conditions of the Proposition 1. we can look for $A(t)$ in different forms. Namely,

Case 1. Let $A(t)=A_{0}(1+t)^{A}$. In this case the conditions of the Proposition 1. are satisfied if and only if

$$
\begin{array}{llll}
0<A_{0}, & 0<\alpha_{0}, & 0<\lambda_{0} & \alpha_{0} \lambda_{0}=1, \\
0<\lambda<A \leq \alpha, & \alpha+\lambda<1, & A \leq 1-\alpha-2 \lambda . &
\end{array}
$$

In general the obtained system of inequalities has lots of solutions, e.g. an appropriate choice of the parameters is

$$
A_{0}=\alpha_{0}=\lambda_{0}=1, \quad A=\alpha=\frac{1}{4}, \lambda=\frac{1}{8} .
$$

Case 2. Let $A(t)=A_{0}(1+t)^{A} \ln (1+t)$. In this case the following inequality system must be satisfied:

$$
\begin{array}{llll}
0<A_{0}, & 0<\alpha_{0}, & 0<\lambda_{0} & \alpha_{0} \lambda_{0}=1, \\
0<\lambda<A<\alpha, & \alpha+\lambda<1, & A<\frac{1}{2}, & A \leq 1-\alpha-2 \lambda .
\end{array}
$$


This system also has solution, e.g.

$$
A_{0}=\alpha_{0}=\lambda_{0}=1, \quad A=\frac{1}{4}, \alpha=\frac{3}{8}, \lambda=\frac{1}{8}
$$

satisfies it.

\section{REFERENCES}

[1] F. Alvarez, "On the minimizing property of a second order dissipative system in Hilbert spaces," SIAM J. Control Optim., vol. 38, no. 4, pp. 1102-1119, 2000.

[2] F. Alvarez and H. Attouch, "An inertial proximal method for maximal monotone operators via discretization of a nonlinear oscillator with damping," Set-Valued Anal., vol. 9, no. 1-2, pp. 3-11, 2001.

[3] A. S. Antipin, "Minimization of convex functions on convex sets by means of differential equations," Differential Equations, vol. 30, no. 9, pp. 1365-1375, 1994.

[4] H. Attouch and R. Cominetti, "A dynamical approach to convex minimization coupling approximation with the steepest descent method," J. Differential Equations, vol. 128, no. 2, pp. 519-540, 1996.

[5] H. Attouch and M.-O. Czarnecki, "Asymptotic control and stabilization of nonlinear oscillators with non-isolated equilibria," J. Differential Equations, vol. 179, no. 1, pp. 278-310, 2002.

[6] H. Attouch, X. Goudou, and P. Redont, "The heavy ball with friction method. I. The continuous dynamical system: global exploration of the local minima of a real-valued function by asymptotic analysis of a dissipative dynamical system," Commun. Contemp. Math., vol. 2, no. 1, pp. 1-34, 2000.

[7] C. A. Botsaris, "Differential gradient methods," J. Math. Anal. Appl., vol. 63, no. 1, pp. 177-198, 1978.

[8] F. H. Branin and S. K. Hoo, "A method for finding multiple extrema of a function of $n$ variables," in Numerical methods for non-linear optimization (Conf., Univ. Dundee, Dundee, 1971), F. A. Lootsma, Ed. London: Academic Press, 1972, pp. 231-237.

[9] A. A. Brown and M. C. Bartholomew-Biggs, "Some effective methods for unconstrained optimization based on the solution of systems of ordinary differential equations," J. Optim. Theory Appl., vol. 62, no. 2, pp. 211-224, 1989.

[10] A. Cabot, "Inertial gradient-like dynamical system controlled by a stabilizing term," J. Optim. Theory Appl., vol. 120, no. 2, pp. 275-303, 2004.

[11] A. Cabot, H. Engler, and S. Gadat, "On the long time behavior of second order differential equations with asymptotically small dissipation," Trans. Am. Math. Soc., vol. 361, no. 11, pp. 59836017, 2009.

[12] A. Cabot, H. Engler, and S. Gadat, "Second-order differential equations with asymptotically small dissipation and piecewise flat potentials," Electron. J. Differ. Equ., vol. 2009, pp. 33-38, 2009.

[13] R. Cominetti, J. Peypouquet, and S. Sorin, "Strong asymptotic convergence of evolution equations governed by maximal monotone operators with Tikhonov regularization," J. Differ. Equations, vol. 245, no. 12, pp. 3753-3763, 2008.

[14] Y. G. Evtushenko and V. G. Zhadan, "Application of the method of Lyapunov functions to the study of convergence of numerical methods," USSR Comput. Math. Math. Phys., vol. 15, no. 1, pp. 96-108, 1975.

[15] A. V. Fiacco and G. P. McCormick, Nonlinear programming: Sequential unconstrained minimization techniques. New York-London-Sydney-Toronto: John Wiley \& Sons, Inc., 1968, vol. XIV. 
[16] S. D. Flåm, "Solving convex programs by means of ordinary differential equations," Math. Oper. Res., vol. 17, no. 2, pp. 290-302, 1992.

[17] X. Goudou and J. Munier, "The gradient and heavy ball with friction dynamical systems The quasiconvex case," Math. Program., vol. 116, no. 1-2 (B), pp. 173-191, 2009.

[18] T. Hajba, "Optimization methods modeled by second order differential equation," Ann. Univ. Sci. Budap. Rolando Eötvös, Sect. Comput., vol. 26, pp. 145-158, 2006.

[19] T. Hajba, "Parameter analysis of optimization methods modeled by second order differential equation," Alkalmazott Mat. Lapok, vol. 26, no. 1, pp. 61-79, 2009.

[20] T. Hajba, "Optimizing second-order differential equation systems," Electron. J. Differ. Equ., vol. 2011, no. 44, p. 16, 2011.

[21] R. Hauser and J. Nedic, "The continuous Newton-Raphson method can look ahead," SIAM J. Optim., vol. 15, no. 3, pp. 915-925, 2005.

[22] M. Kovács, "Continuous analog of gradient-type iterative regularization," Mosc. Univ. Comput. Math. Cybern., vol. 1979, no. 3, pp. 37-44, 1979.

[23] M. Kovács, "Some convergence theorems on nonstationary minimization processes," Math. Operationsforsch. Stat., Ser. Optimization, vol. 15, pp. 203-210, 1984.

[24] A. Nedić, "The continuous projection-gradient method of the fourth order," Yugosl. J. Oper. Res., vol. 5, no. 1, pp. 27-38, 1995.

[25] A. N. Tikhonov and F. P. Vasil'ev, "Methoden zur Lösung inkorrekter Extremalprobleme," Math. Models and numer. Methods, Banach Cent. Publ., vol. 3, pp. 297-342, 1978.

[26] A. Tikhonov, "Solution of incorrectly formulated problems and the regularization method," Soviet Math., Dokl., vol. 5, pp. 1035-1038, 1963.

[27] F. P. Vasil'ev, T. V. Amochkina, and A. Nedich, "A regularized variant of the continuous secondorder gradient projection method," Mosc. Univ. Comput. Math. Cybern., vol. 1995, no. 3, pp. 33-39, 1995.

[28] F. P. Vasil'ev and M. Kovács, "Regularization of incorrectly posed extremal problems using penalty and barrier functions," Mosc. Univ. Comput. Math. Cybern., vol. 1980, no. 2, pp. 30-36, 1980.

[29] F. Vasil'ev and A. Nedich, "The regularized continuous third-order gradient projection method," Differ. Equations, vol. 30, no. 12, pp. 1869-1877, 1994.

[30] F. P. Vasiljev and A. Nedić, "A regularized continuous projection-gradient method of the fourth order," Yugosl. J. Oper. Res., vol. 5, no. 2, pp. 195-209, 1995.

[31] V. I. Venec and M. V. Rybasov, "The method of Lyapunov functions in the study of continuous algorithms of mathematical programming," U.S.S.R. Comput. Math. Math. Phys., vol. 17(1977), no. 3, pp. 64-73, 1978.

[32] L.-H. Zhang, C. T. Kelley, and L.-Z. Liao, "A continuous Newton-type method for unconstrained optimization,” Pac. J. Optim., vol. 4, no. 2, pp. 259-277, 2008.

\section{Authors' addresses}

\section{T. Hajba}

Széchenyi István University, Faculty of Engineering Sciences, Dept. of Mathematics and Computer Science, Egyetem tér 1., H-9026 Győr, Hungary

E-mail address: hajbatesze.hu

\section{Kovács}

Eötvös Loránd University, Dept. of Operations Research, Pázmány sétány 1-3, H-1117 Budapest, Hungary

E-mail address: margoecs.elte.hu 